\title{
HACIA LA UNIVERSIDAD COLABORATIVA Promoviendo un debate sobre el modelo organizativo académico
}

TOWARD COLLABORATIVE UNIVERSITY

Promoting a debate on the academic organization model

\author{
Charles Heckscher checkscher@work.rutgers.edu \\ Center for Organizational Learning \& Transformation. Rutgers University. EEUU.
}

Carlos Martín-Ríos cmartinrios@cminnovation.org

Center for Organizational Learning \& Transformation. Rutgers University. EEUU.

Center for Management Innovation. Madrid. España.

\section{RESUMEN}

El objetivo de este artículo es provocar un debate sobre el futuro de la universidad pública y su modelo de organización. El modelo actual de universidad parece estar en crisis. De los varios factores que contribuyen a esta afirmación, este artículo centra su atención en la idiosincrasia de su modelo organizativo y en las dificultades que éste plantea a la hora de amoldarse a los nuevos retos sociales. Por ello, en los últimos tiempos han aumentado las demandas externas reclamando mayores controles burocráticos que garanticen mayor eficacia en los logros docentes e investigadores. Sin embargo, la consolidación de este modelo supondría sacrificar la capacidad innovadora y de respuesta académica en pos de modelos de trabajo que fomentan menor audacia y mayor uniformidad. A este respecto, se ofrece una propuesta de reformulación de los principios organizativos académicos orientándolos a un modelo de universidad permeable a las necesidades de la sociedad y los diversos grupos de interés a través de la puesta en marcha de prácticas organizativas colaborativas.

\section{Palabras Clave}

Cambio; Organización del trabajo; Profesiones; Universidad colaborativa.

\begin{abstract}
The goal of this article is to promote an open debate on the future of public universities and their organizational model. Current archetype of universities is under question. Though many factors contribute to these strains, this article focuses on the distinctive organization structure of universities and its struggle to adapt to societal challenges. Recently, growing pressures toward higher bureaucratic control on the promise to improve efficiency and productivity are observed. However, the pursuit of the administrative model would lead to a focus on conformity and consistency at the expense of innovation and responsiveness. In that regard, this study offers a proposal for a new organizational system open to stakeholders and the wider society through the adoption of more collaborative organizational practices.
\end{abstract}

KEYWORDS

Collaborative university; Change; Professions; Work organization. 


\section{INTRODUCCIÓN ${ }^{1}$}

En los últimos años se ha entablado un profundo debate sobre los principios que rigen las universidades como organizaciones consignatarias del conocimiento. Más recientemente, a raíz de la crisis económica y el consiguiente cuestionamiento de ciertos paradigmas económicos, políticos y sociales, el debate ha atraído la atención de especialistas de muy diversos ámbitos. En lo que respecta al ámbito académico diversas disciplinas contribuyen ofreciendo una variedad de perspectivas sobre la necesidad de repensar el papel institucional, la viabilidad económica, o el modelo actual de generación y difusión de resultados científicos de la universidad. La gran cantidad de artículos y números especiales sobre estos aspectos así lo acreditan (por ejemplo, Theory and Society, 2011; Science and Public Policy, 2009; o Journal of Management Inquiry, 2012). Estos estudios han puesto de relieve una creciente desafección hacia el modelo actual de universidad como institución, la falta de conexión entre académicos y la sociedad en general, la forma en que los estados financian las universidades públicas, o el impacto actual de la investigación académica. La variedad de temáticas y el calado de las mismas llama a repensar la identidad y forma de trabajar del mundo académico en el siglo XXI (Mayor-Zaragoza 2011; Salaburu 2007).

El presente artículo, de entre los diversos ángulos desde los que analizar el futuro de la universidad, propone abrir un debate sobre la función que los actuales modelos organizativos juegan en la capacidad de respuesta a las necesidades de la sociedad y en la consecución de unos resultados educativos y de investigación acordes a las expectativas. Cimentado en la sociología de las organizaciones y de la educación, este ensayo no pretende realizar una síntesis de la literatura existente sino proponer una serie de argumentos con el fin de provocar un debate enriquecedor sobre el presente y el futuro de las universidades como instituciones de ciencia y educación.

Nuestra tesis es que la actual presión por ofrecer resultados estandarizados, incrementar unos determinados niveles de eficiencia y reducir el exceso de autonomía y, por ende, de ausencia de supervisión sobre el trabajo académico está provocando una revisión del modelo organizativo existente en buena parte de las universidades públicas. En buena medida, la principal demanda es que el modelo presente, profundamente enraizado en los principios gremiales de las profesiones artesanales, deje paso a fórmulas burocráticas de gestión de las tareas académicas esenciales-la investigación y la impartición de docencia-lo cual genera incertidumbre y resistencia entre los académicos. Al tiempo, sugerimos que buena parte de sus normas seculares entorpecen la capacidad de reacción a las demandas externas. El argumento es que las universidades, en tanto que agentes generadores y trasmisores de conocimiento, no pueden vivir de espaldas a las necesidades de la sociedad ni obviar la necesidad de transformarse,

\footnotetext{
${ }^{1}$ Los autores agradecen sus valiosos comentarios a Paul S. Adler.
} 
si bien no necesariamente hacia un modelo administrativo / burocrático como algunos propugnan sino hacia un modelo de organización colaborativa, un concepto que hemos desarrollado en profundidad en otros trabajos (Adler y Heckscher 2013; Adler, Kwon y Heckscher 2008; Heckscher y Martín-Ríos, 2013; Heckscher 2007; Heckscher y Adler 2006).

El presente artículo ofrece un diagnóstico del modelo organizativo universitario dominante, detalla las exigencias de introducción de una organización burocrática descentralizada, sus características y debilidades, y presenta una propuesta para evitar la presión por establecer un mayor control burocrático y avanzar hacia un modelo organizativo más colaborativo, examinando posibles respuestas a partir de los ejemplos de organizaciones que han intentado resolver problemas similares.

\section{LA CRISIS DEL MODELO GREMIAL}

La lógica de las profesiones no ha cambiado excesivamente desde su institucionalización en agrupaciones y gremios artesanales medievales (Adler, Kwon y Heckscher 2008; Freidson, 1994; Abbott, 1988). A saber: los profesionales se inician en actividades con cierto halo de misteriosas, complejas y oscuras que los profanos, entre los que se encuentran los receptores del servicio, no pueden entender. De esta forma, si bien han de rendir cuentas únicamente ante el resto de compañeros de profesión de acuerdo con los códigos deontológicos establecidos, la comunidad confía en su buen hacer dado que en el proceso de aculturación se establece el compromiso de ejercer sus artes anteponiendo el bien de la comunidad y de sus clientes. Los compromisos esenciales del profesionalismo se sustentan en una extensa socialización en los valores y normas de disciplina apropiados del gremio (Sciulli 2005). Más allá de estas instituciones comunitarias autogestionadas, se espera que los profesionales actúen de forma autónoma y juzguen situaciones concretas según su criterio profesional (Cohen y March 1974).

La organización de las universidades ha mantenido esta lógica durante los últimos ocho siglos (Krause 1999; Kerr [1963] 2001). Se ha estructurado alrededor de disciplinas intelectuales autogobernadas, dejando su administración formal, a menudo considerada un mal necesario, relegada a un segundo plano. Los académicos han mantenido unos vínculos disciplinarios muy fuertes, gobernados por la lógica grupal, y han defendido, con gran éxito, su reivindicación de ser los únicos legítimamente capaces de juzgar la calidad y el valor de su labor (Cohen y March 1974).

Hace un siglo y medio este modelo gremial prevalecía en toda la sociedad, el cual caracterizaba no solamente a las principales profesiones sino a las agrupaciones de artesanos en general. Artesanos de todo tipo —desde tipógrafos a sopladores de vidrio - comenzaban su labor como aprendices para ir instruyéndose en los misterios de sus oficios que, en gran medida, se autorregulaban mediante las estructuras gremiales (Waters 1989; Richardson 2001). Sin embargo, uno tras otro, estos oficios 
fueron sucumbiendo a una lógica distinta, a un modelo más "moderno" que combina el modelo organizativo burocrático con la lógica del mercado (Kritzer 1999; Haug, 1973; Hall 1968). No fue ésta una transición sencilla. Con frecuencia los gremios resistieron ferozmente, incluso en ocasiones de forma violenta, a la intromisión de agentes externos en su modo de operar tradicional (Leicht y Fennell 1997). Pero, tal y como Weber predijo, este enfoque "racional" moderno ha demostrado ser más eficaz y mejor en muchos aspectos. El modelo burocrático operando dentro de la lógica del mercado ha mostrado su capacidad para alcanzar unos niveles de producción más elevados, unos estándares de calidad mayores y más constantes, un enorme ahorro en cuanto a costes y un mayor grado de innovación en comparación con el modelo tradicional al que sustituían. Con el tiempo esta lógica administrativa se ha extendido incluso a las profesiones más técnicas e intensivas en conocimiento, tales como las ingenierías (Layton 1971) o la informática (Cusumano 1992).

Los académicos, junto con los abogados y los médicos, constituyen los últimos representantes significativos de los antiguos oficios autogobernados. Ahora bien, hoy en día estos grupos se encuentran inmersos en la misma guerra que sus predecesores y aparentemente la están perdiendo (Pfadenhauer 2006; Halpern 1992; Rothman 1984). Por ejemplo, la presión que se está ejerciendo sobre la práctica médica está particularmente bien documentada (Ritzer y Walczak 1988; Scott 1982). Por parte de la "demanda", se observa de forma general que la deferencia de los pacientes al juicio profesional ha disminuido a lo largo de las últimas décadas, una tendencia propiciada en parte por el aumento de información disponible en Internet, a la que es fácil acceder (Fintor 1991; Landzelius 2006). Existe una creciente demanda de información respecto a la actuación de los médicos como mecanismo de rendición de cuentas (accountability) ante los pacientes. Al tiempo, los vínculos personales entre médico y paciente se han ido debilitando. Como apunta Kuhlmann (2006), la atención sanitaria es cada vez más "incorpórea", está menos basada en la confianza personal y más en la veracidad de la información suministrada. Esta tendencia se ve reflejada en el aumento de pleitos judiciales por mala praxis y la aceptación gradual en los juzgados de casos de práctica médica (Peters 2000). Por parte de la "oferta", la asistencia médica resulta cada vez más compleja al requerir, por una parte, una necesidad de coordinación entre múltiples especialidades y tecnologías y, por otra, una erosión de los modelos tradicionales de autonomía. Ahora bien, estudios recientes apuntan a una mayor percepción negativa de la ciudadanía sobre la independencia médica y la calidad de la asistencia sanitaria; al tiempo que otra línea de investigación ha documentado la relación que se establece entre la ausencia de regularización (siquiera de ciertas prácticas básicas como lavarse las manos) y el incremento en la accidentalidad, incluso muertes de pacientes (Gawande 2007). La defensa que hacen los médicos de su sistema de autogobierno se ha visto menoscabada por la debilidad de la disciplina profesional (Feinstein, Gutkind y Verghese 2007).

Aunque el mundo académico no ha evolucionado exactamente igual —no se nos ha relacionado en exceso con la mala praxis- la mayor parte de profesores reconocerían 
la misma dinámica inherente por lo que respecta a sus dos actividades esenciales: la docencia y la investigación (Waters 1989). Así, los estudiantes (y sus padres) muestran cada vez menos deferencia al criterio profesional y exigen mayores justificaciones en las evaluaciones, las calificaciones y los requisitos para obtenerlas. En paralelo, las evaluaciones de calidad docente por parte de los estudiantes son cada vez más populares, alcanzando una relevancia notoria con una rapidez sorprendente. En segundo lugar, al igual que se ha producido una considerable "invasión" de la autonomía curricular de los maestros de primaria y secundaria por parte de las juntas directivas escolares y de los organismos oficiales, lo mismo está empezando a suceder en la universidad. Bajo unos determinados criterios de eficiencia y calidad docente, se argumenta que la educación universitaria requiere disciplina curricular. Asimismo, la estructura tradicional académica comienza a parecer ineficaz e ineficiente entre determinados colectivos. Entre las principales quejas de los estudiantes cabe reseñar la falta general de interés en la docencia por parte de los académicos: observan excesiva independencia y desconexión entre los profesores universitarios y consideran que algunos se preocupan por enseñar y otros no. Asimismo, el hecho de enfatizar la independencia académica apunta a la percepción de una ausencia notable de coordinación entre las materias impartidas (Salaburu 2007).

Por lo que respecta a la investigación académica, dada la casi exclusiva atención en la evaluación por parte de pares, buena parte de los trabajos de investigación sigue siendo desconocida e inaccesible para el gran público, por lo que han acrecentado las reiteradas quejas acerca de la escasa relevancia y la ausencia de beneficios tangibles de los resultados ofrecidos (Hitt y Greer, 2012). Las agencias estatales financiadoras reprochan que los resultados de investigación no son proporcionales a la cantidad de fondos invertidos; determinados colectivos y medios de comunicación critican la dispersión de la actividad investigadora (Bloom y Bellow 1988); desde el ámbito empresarial se desaprueba la escasa atención a la aplicabilidad y la obtención de beneficios financieros (Brock, Hinings y Powell 1999); incluso desde el propio entorno académico se critica la cantidad de trabajos de investigación. Resulta pertinente la crítica realizada por el catedrático en economía Vedder (2011) quien se lamentan los 21.000 artículos que estima se han publicado sobre Shakespeare desde 1980 y se pregunta si no hubiera sido suficiente con 5.000. Entretanto, la creciente presión hacia mayores niveles de productividad (resultados) en la investigación lleva a muchos académicos a reducir la carga lectiva (Clark 2006; Boyer 1997).

Así las cosas, aumenta la sensación de que las principales actividades de la profesión tienen cada vez menos que ver con las demandas de la sociedad, a lo que contribuye la idea, extendida interesadamente por ciertos colectivos, de que el mundo académico se mantiene al margen de la creciente preocupación social por la contención de costes y la generación de valor. Como señala Unceta "la universidad tiene su propia forma de hablar y también su particular visión de la historia y del papel social que le corresponde desarrollar, en definitiva su cultura" (2011: 1122). La fuerte cultura, la forma de funcionamiento y la historia de la profesión obstaculizan la posibilidad de enmendar la situación: los académicos se sienten acosados e incomprendidos, mientras que los 
colectivos directa e indirectamente implicados con el mundo académico se sienten cada vez más alienados e ignorados (Fernández Enguita 2009).

\section{LA ALTERNATIVA ADMINISTRATIVA / BUROCRÁTICA. UN MAYOR CONTROL}

En las últimas décadas, diversos expertos y grupos de interés o stakeholders han comenzado a propugnar acciones contundentes para traspasar los muros del cerrado mundo universitario. Parte de las demandas abogan por desarticular la forma organizativa tradicional y, con la promesa de mejorar la eficiencia y la capacidad de respuesta, implantar modelos de gestión burocráticos 0 administrativos de la labor académica. Es lo que autores como Bousquet (2008) denominan la "corporization" de las universidades, trasladar al ámbito académico determinados modelos de gestión empresarial con el objetivo, más o menos manifiesto, de ejercer un mayor control sobre las prácticas académicas, que como hemos reflejado anteriormente a menudo parecen solipsistas y autocomplacientes.

Si simplemente transfiriéramos el conocimiento organizativo adquirido por las grandes corporaciones en un siglo de desarrollo, obtendríamos una estructura jerárquica centrada en torno a indicadores de eficiencia, coherencia y control. Habría una clara diferenciación entre funciones: por una parte, profesionales de la enseñanza y, por otra, investigadores. Así, por ejemplo, los primeros estarían regulados por estrategias y programas docentes coordinados y su responsabilidad se ceñiría a alcanzar unos determinados objetivos de aprendizaje. Dichos objetivos se centrarían en las necesidades y deseos de los estudiantes, transmitidos por un mercado libre que conferiría la capacidad de libre elección a los consumidores (familias) y obligaría al mundo académico a satisfacer dichas necesidades en lugar de ceñirse a la concepción tradicional de las áreas de conocimiento autodefinidas. La autoridad administrativa impondría unos estándares de calidad y unos indicadores de eficiencia de la docencia impartida.

De hecho, este modelo es cada vez más común en la educación. Por ejemplo, en los niveles preuniversitarios norteamericanos, iniciativas educativas como "No Child Left Behind" o "Race to the Top" han impuesto precisamente este tipo de estandarización y responsabilidad administrativas. En España y otros países europeos la creciente implantación de los rankings escolares pretende equiparar padres y consumidores a través de mecanismos del mercado. Las universidades, por su parte, ya participan de un sector "experimental" dominado por instituciones educativas privadas, las cuales están ganando cierta relevancia con respecto a sus homólogos públicos; y sus "consumidores" son cada vez más asertivos. Otras iniciativas apuntan a la creciente estandarización de las actividades escolares. Por ejemplo, en el año 2006 el Departamento Federal de Educación norteamericano propuso crear una base de datos pública de medidores del rendimiento que permitiera a los padres estar mejor informados a la hora de tomar decisiones, aumentando de esta forma la obligación escolar de rendir cuentas (Spellings 2006). En 
Gran Bretaña, el Plan de Excelencia en Investigación (Research Excellence Framework) pretende basar explícitamente la inversión pública en el impacto y la relevancia social de las iniciativas académicas (Higher Education Funding Council for England 2011). En España, la creación de una red de campus de excelencia internacional o los sistemas homologados de acreditación académica y del profesorado regulados por la Agencia Nacional de Certificación Académica (ANECA) siguen pautas similares. Más en general, las clasificaciones realizadas por U.S. News \& World Report, Times Higher Education, Financial Times y otros medios de comunicación tienen tal repercusión que a menudo sus resultados sirven de base para la concesión de premios o, en casos específicos, para justificar la necesidad de reestructuraciones.

Otros indicadores avalan la tesis de una creciente formalización en las labores académicas. Así, por ejemplo, se ha extendido la práctica de valorar a los académicos basándose en la lógica fordista de gestión por objetivos que equipara la calidad y el valor con una producción eficiente (medida de manera burda por la cantidad de docencia impartida y el número de publicaciones alcanzado). En esa línea, el número de profesores adjuntos (profesionales contratados para tareas exclusivamente docentes, ajenos a la lógica de promoción y titularidad y con condiciones laborales sensiblemente peores a las de los profesores de carrera) se ha disparado en las últimas décadas (Baldwin y Chronister 2001; JBL Associates 2008), mientras que los académicos tradicionales han reenfocado su actividad hacia tareas relacionadas con la publicación de trabajos de investigación, reduciendo en la medida de lo posible su dedicación a la enseñanza (Basken 2011; Higher Education Academy 2009).

Todos estos acontecimientos reflejan la combinación esencial de mercado y control burocrático que caracteriza a la sociedad moderna y que se está apoderando con fuerza de determinados aspectos académicos que tradicionalmente habían permanecido autogestionados.

\section{UNA ALTERNATNA A LOS MODELOS ESTANDARIZADOS. LOS SISTEMAS COLABORATIVOS}

Es poco probable que las universidades resistan la presión para que se ejerza un mayor control burocrático sobre ellas arguyendo su antigua reivindicación de ser los únicos expertos indiscutibles. Sería mejor que miraran a su alrededor y se fijaran en alternativas organizativas implementadas por determinadas empresas interesadas en buscar soluciones a las ineficiencias provocadas por los modelos burocráticos tradicionales (Heckscher y Donnellon 1994). Y si lo hicieran observarían una extraña paradoja: mientras que el control burocrático está llamando a la puerta y salvando el escollo de las profesiones, ese mismo modelo burocrático recibe cada vez más críticas y sufre más modificaciones en algunas de las empresas más innovadoras. Como buena parte de la investigación actual pone de relieve, algunas empresas de gran éxito promueven diseños organizativos que requieren menos control jerárquico y más trabajo en equipo. 
$\mathrm{Si}$, como la literatura ha mostrado extensamente, el modelo burocrático tradicional provoca innumerables disfunciones e ineficiencias tanto en el gobierno como en las empresas, ¿no deberíamos preguntarnos si vale la pena aplicarlo a las universidades?

Cabe prestar atención a las innovaciones organizativas llevadas a cabo en algunas de las grandes corporaciones globales, como es el caso de IBM, otrora arquetipo tradicional de un modelo administrativo, altamente burocrático y bien organizado, centrado en producir equipos y venderlos en el mercado. Dos fenómenos desencadenaron la necesidad de cambio: los mercados se hicieron más inestables y complejos debido a los cambios tecnológicos, a la globalización de los mercados y a la mayor sofisticación de los consumidores; y el proceso de producción se hizo más exigente, requiriendo altos niveles de flexibilidad y constante innovación con los que combinar esferas del conocimiento operativo y de gestión anteriormente muy distantes entre sí (Applegate, Heckscher, Boniface y Collins, 2003).

En este contexto, tal modelo administrativo demostró ser ineficaz a la hora de dar respuesta a las necesidades del momento. Un problema podía enquistarse durante años antes de que los verdaderos responsables de la toma de decisiones se ocuparan de resolverlo. De hecho, es lo que sucedió en la década de los 90 cuando los equipos de ventas de IBM hacía años que sabían que el PC se estaba convirtiendo en una amenaza para el modelo empresarial de la compañía, pero la organización en su conjunto no reaccionó hasta que la gente, bastante repentinamente, dejó de comprar ordenadores de IBM, con la consiguiente crisis financiera e institucional. Internamente, la resolución de esta crisis pasaba por la participación en la toma de decisiones de cargos y departamentos acostumbrados a trabajar de manera autónoma. Éstos, actuando según los preceptos organizativos burocráticos, descargaban responsabilidades en sus superiores; de forma que cada vez las decisiones se debían tomar en niveles más altos de la jerarquía obligando a una creación y revisión constante de las normas lo que llegó a provocar que el engranaje dejara de funcionar casi por completo.

La reacción a esta crisis, no obstante, no supuso volver al modelo organizativo gremial del siglo XIX. Antes al contrario, supuso un giro lento y difícil hacia un tercer modelo, ni gremial ni burocrático, sino colaborativo (Applegate et al. 2003). Una organización colaborativa (en corporaciones) es aquella en la que los clientes están en contacto con ella de forma continuada y en la que los departamentos de las distintas especialidades trabajan juntos mediante grupos de trabajo en lugar de esperar a que se creen normas administrativas (Adler, Heckscher y Prusak 2011; Heckscher y Adler 2006). Su principio rector no es la autonomía, sino la interdependencia, pues pretende reubicar a los especialistas en procesos coordinados. Ha demostrado ser capaz de movilizar rápidamente conocimientos diversos y distantes para resolver problemas complejos y responder con prontitud a los cambios en lo que respecta a las expectativas de los clientes.

Internamente, IBM y otras compañías - desde Procter \& Gamble a Toyota e incluso, en parte, GM y Shell Oil— han dedicado considerable esfuerzo en las últimas décadas a reducir la burocracia e idear sistemas colaborativos (Heckscher 2007). Algunas de ellas han desarrollado robustas redes de equipos y grupos de trabajo que colaboran por 
encima de las habituales fronteras burocráticas con el fin de reunir las mejores aptitudes para resolver cualquier problema que surja. Se trata de un problema organizativo complicado, cuya puesta en marcha en ocasiones provoca resistencia por parte de los gerentes y de determinados empleados. Sin embargo, provee soluciones a algunos de los problemas más graves que acucian a las empresas tradicionales (Sabel 2006). Muchas de ellas se han visto muy perjudicadas por la rigidez del modelo de compartimentos funcionales estancos y demás divisiones internas, organizadas sobre los principios burocráticos y han conseguido superarlo en parte básicamente gracias a dos cambios.

En primer lugar, se han esforzado mucho en delinear un propósito común. En los últimos años se ha producido un interés generalizado porque los empleados en todos los niveles de la compañía discutan de forma abierta y continuada las estrategias. Se crea así un punto de referencia común que trasciende las culturas funcionales particulares y motiva a la gente para que aplique su experiencia profesional en la consecución del propósito general (Kaplan y Norton 2001; Coleman 1993).

El segundo cambio ha sido la consolidación de equipos de trabajo interdisciplinares y flexibles (Scott 2001). Hoy en día en muchas compañías los cargos intermedios y los empleados técnicos pasan gran parte de su tiempo trabajando no bajo la dirección de sus jefes formales, sino en proyectos que requieren la intervención de especialistas de diversas áreas funcionales. Esto comporta cambios en la cultura organizativa, así como en principios de responsabilidad y especialidad laboral; pero a resultas de estos cambio se consigue movilizar distintos conocimientos para la resolución de problemas complejos. Desarrollar un producto, por ejemplo, era antes una tarea que se realizaba en un proceso lineal, desde el diseño hasta la ingeniería, la producción y las ventas. El producto pasaba de un departamento a otro en cada etapa del proceso y a menudo regresaba al departamento anterior si surgían problemas. Este proceso arduo y poco eficaz se ido sustituyendo por un diseño e ingeniería simultáneos, en los que diversos especialistas de cada función concreta se reúnen en una misma mesa y resuelven los problemas continuadamente a medida que aparecen (Clark et al. 1987; Donnellon 1993; Galbraith 1994).

Externamente, estas mismas compañías (desde IBM a P\&G y Apple) están creando sistemas para poder interactuar más y de una forma más continua con sus clientes. Tratan de incluir a los clientes no solamente en grupos de discusión relacionados con estrategias de publicidad, sino en la propia toma de decisiones en cuanto al diseño y desarrollo del producto (French, LaBerge y Magill 2011; Grant 2011). La filosofía que se está imponiendo poco a poco en estas grandes empresas se basa en tratar de crear soluciones en lugar de productos, es decir, dedicarse a comprender qué necesitan realmente los clientes y tratar de satisfacerlos. Así, una de las recientes revisiones de los valores centrales de IBM, que surgió a partir de un intenso proceso de discusión interna, ha dado lugar a la llamada "dedicación al éxito de cada cliente". Este nuevo valor implica un cambio de mentalidad bastante revolucionario: mientras que antes se centraban en mejorar la oferta de productos basándose en criterios técnicos definidos internamente, el nuevo enfoque comporta una relación interactiva en la que prioridades enfrentadas -los 
estándares técnicos y las necesidades de los clientes- "discuten" entre sí con el fin de incorporar lo esencial de cada una (Galbraith 2002). Además, este método interactivo 0 de búsqueda de soluciones se está extendiendo a otras partes implicadas aparte de los clientes, con lo que se está reconociendo que es vital que haya un mayor apoyo social para el buen funcionamiento de las empresas a largo plazo (Sabel 2006).

\section{¿SON POSIBLES LAS UNIVERSIDADES COLABORATIVAS?}

Así pues, paradójicamente, las mismas corporaciones que nos han mostrado los puntos negativos de la gestión burocrática también han empezado a mostrar los elementos de una alternativa. La historia de GM y de IBM pone de manifiesto las rigideces que se producirán si se aplica un modelo burocrático / administrativo a este ámbito: es probable que se dilapiden la innovación y la capacidad de reacción características de la organización profesional. El mundo académico puede aprender de ambos aspectos en esta experiencia. El modelo de colaboración que estas compañías están explorando puede resultar apropiado para el ámbito académico, si bien teniendo en cuenta los modos tradicionales de funcionamiento su implantación no resulte necesariamente sencilla.

Sin duda, consolidar un modelo administrativo llevaría a las universidades, al igual que a las empresas, a una burocratización en el sentido negativo de la palabra, basada en la conformidad y la coherencia a expensas de la innovación y la capacidad de reacción. Tal y como mostramos en los ejemplos precedentes, a medida que aumentan los controles administrativos, las grandes corporaciones jerárquicas pierden sistemáticamente de vista las necesidades de los clientes, limitando la capacidad de comprender dichas necesidades y de responder a ellas. En ese contexto, seguir las reglas establecidas se convierte en la norma y la gente tiende inevitablemente a centrase en hacer su trabajo tal como se exige desde los ámbitos de decisión. Como se apuntó anteriormente, esta tendencia ya se puede ver en los programas educativos más burocratizados, donde los profesores, sujetos a una excesiva normalización, tienden a "enseñar para aprobar el examen" e intentan alcanzar unos objetivos que los apartan de la interacción real con sus alumnos. De un modo semejante, la extendida tendencia a conceder subvenciones y ayudas a la investigación en función de una supuesta relevancia social conmensurable fomenta el que los académicos centren su labor en la obtención de resultados a corto plazo (presentaciones, publicaciones), dejando de lado tareas de investigación a largo plazo de carácter más incierto.

¿Pueden aprender las universidades de los cambios positivos en la organización del trabajo que están introduciendo las corporaciones colaborativas? A favor del modelo colaborativo tenemos que este tipo de enfoque es más propicio para la producción y el intercambio de conocimientos e interconectaría a los académicos en mayor medida que un modelo administrativo, burocrático. En su contra está el hecho de que se aleja bastante de la orientación tradicional de autonomía y autogobierno profesionales y requeriría cambios de gran envergadura. 
En lo que respecta a la toma de decisiones interna, un sistema colaborativo implicaría la interacción entre académicos de líneas disciplinares distintas para hacer frente a los retos comunes. Esto supondría invertir mucho más tiempo del que pasan ahora asistiendo a comités interdepartamentales, considerando los puntos de unión y flujos entre disciplinas tal como las perciben los estudiantes y las demás partes implicadas, y creando ofertas conjuntas que tuvieran en cuenta los intereses emergentes y las necesidades sociales. Hasta el momento han sido principalmente las administraciones académicas las que han intentado crear dicha colaboración interdepartamental encontrándose con grandes obstáculos en su empeño. Los académicos, por su parte, más centrados en sus disciplinas, han mostrado poco entusiasmo en el mejor de los casos. Las grandes diferencias entre las diversas tradiciones académicas dificultan que se produzca una auténtica comunicación entre los distintos departamentos.

Pongamos un ejemplo típico. Nadie duda de la creciente importancia de China en la economía mundial. En general, se considera positivo conocer más a fondo la cultura china. Es una opinión social generalizada, sobre todo entre estudiantes y empresas. Los departamentos de economía y administración de empresas han tratado de abordar esta necesidad sin dilación ofertando cursos y postgrados específicos (uno de los motivos por los que estas disciplinas son famosas es por su rápida capacidad de respuesta a cambios en la demanda, como lo demuestra el desarrollo constante de programas en temáticas variadas como la gestión de recursos humanos, el emprendimiento social, o el marketing digital y las redes sociales). En la estructura actual, los departamentos de administración de empresas intentan asumir la responsabilidad de ofertar dichos cursos de forma independiente, esforzándose en que el profesorado desarrolle nuevas competencias. Cabe plantearse si es el enfoque óptimo. En general, dichos académicos carecen de un conocimiento profundo de la cultura, la sociedad y el idioma chinos, de modo que sencillamente tienden a reproducir la metodología, manuales y ejercicios elaborados en otros países. Desde el enfoque colaborativo, sería más apropiado que se asociaran con los departamentos de idiomas, sociología, historia e incluso arte chinos con el objetivo de preparar un currículo que verdaderamente integrara el cuerpo de conocimiento existente. Seguramente tal colaboración otorgaría a estos últimos una nueva base para su legitimación y apoyo, al tiempo que mejoraría la calidad de los resultados que se obtuvieran.

Sin embargo, con ejemplos como éste se hacen más evidentes los obstáculos. Cada disciplina habla literalmente idiomas distintos, difieren en cuanto a prioridades, tradiciones de publicación, tipos de estudiantes y normas internas. Crear algo que combinara esas bases de conocimiento requería un esfuerzo y un compromiso de colaboración muy alto; $y$ hacerlo de manera que no perjudicase las perspectivas laborales y de promoción del personal académico sería aún más difícil. ¿Cómo asignar "crédito" a esas tareas, tanto en la categoría de servicio como en la de investigación? ¿A quién se le concedería el dinero proveniente de las matrículas? ¿Cómo se tomarían las decisiones? Estas preguntas tienen difícil respuesta tanto desde un prisma burocrático / administrativo como desde el marco tradicional. 
Ciertamente, la lógica colaborativa ha ganado terreno en el ámbito de la investigación. El proceso de investigación académica se ha convertido en muchos campos en una tarea multidisciplinar formada por equipos de académicos. Donde hace tan solo unas décadas el "patrón oro" eran trabajos realizados por un único autor, hoy día es habitual que los artículos en ciencias sociales y humanidades cuenten con dos o tres autores e, incluso más en el caso de las ciencias "duras" ( Jones, Wuchty y Uzzi 2008; Manton y English 2007; Shaban 2007). Esta transformación genera tensiones en los modelos tradicionales de evaluación por parte de expertos pues resulta más complejo asignar la titularidad de los méritos. También está reñido con los mecanismos burocráticos actuales y el deseo subyacente de estandarizar los resultados de la investigación. Así, en el Reino Unido, en España y en otros países europeos, los sistemas de acreditación empleados en normalizar la carrera (titularidad y promoción) y retribución de los académicos penalizan las publicaciones en las que participan varios autores.

La tarea más difícil de todas, la cual parece estar muy alejada del modo de obrar habitual de los académicos, es la necesidad de involucrar en las discusiones sobre el propósito de la universidad a todos aquellos colectivos externos interesados. Los académicos raras veces intercambian opiniones sobre el valor de su trabajo fuera del ámbito académico. Fuertemente arraigados en su cultural gremial, corporativista, se limitan a asumir y a creer que lo que hacen es importante. De hecho, la posibilidad de exigir que se justifique la trascendencia de su trabajo supone una amenaza en sí misma por cuanto implica la posibilidad de que profanos en la materia puedan entrometerse en el funcionamiento interno académico. Sin embargo, la base sobre la que debe asentarse el modelo colaborativo descansa en una definición consensuada del propósito común a través de la participación en las discusiones de todas las partes interesadas. Para ello, las universidades deberían revelar los misterios de la profesión y el propósito de las diversas disciplinas académicas. Por una parte, ciertas disciplinas (por ejemplo, la literatura medieval francesa en alusión a Vedder) deberían justificar su relevancia a pesar de aparente falta de acomodo. ¿Qué criterios sigue, en qué se basa para merecer el apoyo social? ¿Qué bien hace, en qué contribuye a la sociedad y qué aporta a los estudiantes? Por otra parte, también deberían cuestionarse aquellas otras disciplinas como la economía, las ingenierías o la administración de empresas a pesar de gozar de una cierta popularidad. ¿Realmente añaden valor en cuestiones tales como la carrera profesional de los estudiantes, los estándares de bienestar o la calidad de vida de las personas? Tales discusiones forzarían al ámbito académico a justificar su aportación a una serie de principios y valores que incluirían la preservación de tradiciones intelectuales; pero también su función en la salvaguardia de una sociedad civil diversa o la prosperidad económica. Este ejercicio supondría entablar una conversación abierta y honesta para convencer al resto de partes interesadas de que vale la pena invertir en estos valores. 


\section{CONCLUSIONES}

La primera conclusión de este ensayo es que existe un gran problema: las universidades necesitan una reorganización profunda porque han perdido su capacidad de reacción ante las instituciones y agentes que les proporcionan los recursos y, por tanto, también han perdido en parte su confianza. La segunda conclusión es que es probable que la reforma actual más popular, asociada a un mayor control administrativo y una mayor burocratización, empeore las cosas para todos aquellos implicados, alumnos, académicos, las propias universidades y la sociedad en general. Y la tercera es que vale la pena fijarse en los cambios recientes en cuanto a modelos organizativos introducidos en determinadas empresas para poder vislumbrar cómo podría ser la alternativa.

Puede parecer paradójico argumentar que algunas corporaciones, a menudo conocidas por su inercia burocrática, pueden ser un ejemplo válido de cómo evitar los problemas derivados de la burocratización. Nuestro planteamiento es que, precisamente algunas de esas empresas ya se han lanzado a superar las imposiciones organizativas burocráticas y, por tanto, han comenzado a abrir un sendero hacia un destino distinto. Han comenzado a mostrar que una organización colaborativa es una alternativa factible a la organización burocrática, una organización que sí puede reaccionar ante las necesidades de los stakeholders sin limitarse a obedecer simplemente a las demandas del mercado a corto plazo, que puede involucrar a los clientes y a las demás partes interesadas en un verdadero diálogo acerca de los objetivos y que también es eficaz a la hora de alcanzar dichos objetivos.

Quizás haga falta una auténtica crisis para superar los siglos de aculturación que llevamos a nuestras espaldas. Es posible que la actual crisis en la que estamos inmersos actúe como revulsivo y surjan iniciativas que dinamicen las bases actuales de la universidad. $Y$ es bastante probable que la resistencia académica al cambio y la insistencia en la especialización autónoma sean tan fuertes que la cuerda se rompa antes de ceder; que la sociedad en general, al no tener una excesiva motivación a involucrarse con el profesorado, deje en gran medida de mostrarle su apoyo. Empresas como IBM y GM han pasado por este tipo de crisis, perdiendo el contacto con sus usuarios y clientes y, por ello, casi desapareciendo en el proceso. Quizás las universidades públicas han comenzado a experimentarlo ahora. La crisis económica ha acelerado la necesidad de entablar este debate. Aquellos de nosotros que estamos en universidades públicas conocemos bien la tendencia de los departamentos a proteger sus propios recursos y tradiciones en lugar de involucrarse en discusiones aparentemente desordenadas, desagradables, largas y frustrantes con aquellos que no les entienden.

La organización colaborativa ha demostrado poder ofrecer unos resultados positivos, una capacidad para movilizar conocimientos diversos, para responder a los cambios complejos del entorno y para involucrar a múltiples partes interesadas. El camino para llegar a ella es largo y arduo, pero en nuestra opinión es la mejor, quizás la única, alternativa disponible. 


\section{REFERENCIAS BIBLIOGRÁFICAS}

Abbott, A. 1988. The System of Professions: An Essay on the Division of Expert Labor. Chicago: University of Chicago Press.

Adler, P. S. y C. Heckscher. 2013. "Collaborative ambidextrous organizations ". Universia Business Review 40: 34-51.

Adler, P. S., C. Heckscher y L. Prusak. 2011. "Building a collaborative enterprise". Harvard Business Review 95-101.

Adler, P. S., S. W. Kwon y C. Heckscher. 2008. "Professional work: The emergence of collaborative community". Organization Science 19: 359-376.

Applegate, L., C. Heckscher, M. Boniface y E. Collins. 2003. "IBM's decade of transformation: Uniting vision and values". 9-807-030. Boston: Harvard Business School University Publishing.

Baldwin, R. G. y J.L. Chronister. 2001. Teaching without Tenure: Policies and Practices for a New Era. New York: Johns Hopkins University Press.

Basken, P. 2011. "Scientists fault universities as favoring research over teaching". The Chronicle of Higher Education, 13 de enero.

Bloom, A. y S. Bellow. 1988. The Closing of the American Mind. New York: Simon \& Schuster.

Boyer, E.L. 1997. Scholarship Reconsidered: Priorities of the Professoriate. San Francisco, CA: JosseyBass.

Brock, D., C. R. Hinings, y M. Powell. 1999. Restructuring the Professional Organization: Accounting, Health Care, and Law. London, UK: Routledge.

Clark, W. 2006. Academic Charisma and the Origins of the Research University. Chicago: University of Chicago Press.

Clark, K. B., W. B. Chew, T. Fujimoto, J. Meyer y F. M. Scherer. 1987. "Product development in the world auto industry". Brookings Papers on Economic Activity 729-781.

Cohen, M.D. y J.G. March. 1974. Leadership and Ambiguity: The American College President. New York: Mc Graw Hill.

Coleman, J.S. 1993. "Social organization of the corporation". Pp. 172-190 in Ethical Issues in Business: A Philosophical Approach. Englewood Cliffs, NJ: Prentice Hall.

Cusumano, M. A. 1992. "Shifting economies: From craft production to flexible systems and software factories". Research Policy 21: 453-480.

Donnellon, A. 1993. "Crossfunctional teams in product development". Journal of Product Innovation Management 10: 377-392.

Feinstein, K. W., L. Gutkind y A. Verghese. 2007. Silence Kills: Speaking Out and Saving Lives. Dallas, TX: Southern Methodist University Press.

Fernández Enguita, M. 2009. "El anti-Cándido: Todo va mal pero irá a peor. Alcance y funciones de la visión apocalíptica de la enseñanza por el profesorado. Papeles de Economía Española 59-68. 
Fintor, L. 1991. "Patient activism: Cancer groups become vocal and politically active". Journal of the National Cancer Institute 83: 528 -529.

Freidson, E. 1994. Professionalism Reborn: Theory, Prophecy, and Policy. Chicago: University of Chicago Press.

French, T., L. LaBerge y P. Magill. 2011. "We're all marketers now”. McKinsey Quarterly julio.

Galbraith, J.R. 1994. Competing with Flexible Lateral Organizations. Reading, Mass: Addison-Wesley.

Galbraith, J.R. 2002. "Organizing to deliver solutions". Organizational Dynamics 31: 194-207.

Gawande, A. 2007. "Annals of medicine: The checklist: reporting \& essays". The New Yorker, 10 de diciembre.

Grant, A. M. 2011. "How customers can rally your troops". Harvard Business Review 89: 96-103.

Hall, R. H. 1968. "Professionalization and bureaucratization”. American Sociological Review 33: 92-104.

Halpern, S. A. 1992. "Dynamics of professional control: Internal coalitions and crossprofessional boundaries". American Journal of Sociology 97: 994-1021.

Haug, M. 1973. "Deprofessionalization: An alternative hypothesis for the future". Sociological Review Monograph 20: 195-211.

Heckscher, C. 2007. The Collaborative Enterprise. New Haven, CT: Yale University Press.

Heckscher, C. y P. Adler. 2006. The Firm as a Collaborative Community: Reconstructing Trust in the Knowledge Economy. New York: Oxford University Press.

Heckscher, C. y C. Martin-Rios. 2013. "Looking back, moving forward: Toward collaborative universities". Journal of Management Inquiry 22(1): 136-139.

Heckscher, C., A. Donnellon, eds. 1994. The Post-Bureaucratic Organization. Oxford University Press, New York.

Higher Education Academy 2009 Reward and Recognition of Teaching in Higher Education: A Collaborative Investigation. http://www.heacademy.ac.uk/assets/documents/resources/publications/ Reward_and_Recognition_Interim.pdf, último acceso el 20/04/2012.

Higher Education Funding Council for England. 2011. HEFCE : Research Excellence Framework. http:/l www.hefce.ac.uk/research/ref/ , último acceso el 20/04/2012.

Hitt, M. y C. Greer 2012. The value of research and its evaluation in business schools: Killing the goose that laid the golden egg? Journal of Management Inquiry 21: 236-240.

JBL Associates. 2008. Reversing Course: The Troubled State of Academic Staffing and a Path Forward. for the American Federation of Teachers. http://www.aft.org/pdfs/highered/reversingcourse1008. pdf, último acceso el 20/04/2012.

Jones, B., S. Wuchty, y B. Uzzi. "Multi-university research teams: Shifting impact, geography, and stratification in science". Science otoño.

Kaplan, R.S. y D.P. Norton. 2001. "Transforming the balanced scorecard from performance measurement to strategic management: Part II". Accounting Horizons 15: 147-160. 
Krause, E. A. 1999. Death of the Guilds: Professions, States, and the Advance of Capitalism, 1930 to the Present. New Haven, CT: Yale University Press.

Kritzer, H. H. 1999. "The professions are dead, long live the professions: Legal practice in a postprofessional world". Law \& Society Review 33: 713-759

Kuhlmann, E. 2006. "Traces of doubt and sources of trust: health professions in an uncertain society". Current Sociology 54: 607-620.

Landzelius, Kyra. 2006. "Introduction: Patient organization movements and new metamorphoses in patienthood". Social Science \& Medicine 62: 529-537.

Layton, E. T. 1971. The Revolt of the Engineers. Social Responsibility and the American Engineering Profession. Baltimore, MD: Johns Hopkins University Press.

Leicht, K. T., M. L. Fennell. 1997. "The changing organizational context of professional work". Annual Review of Sociology 23: 215-231.

Manton, E. y D. English. 2007. "The trend toward multiple authorship in business journals". Journal of Education for Business 82: 164-168.

Mayor-Zaragoza, F. 2011. "The university of the 21st century, political and social trends of globalization: Challenges for higher education". Higher Education in the World: New Challenges and Emerging Roles for Human and Social Development pp. 20-27,

Peters, P.G. 2000. "Quiet demise of deference to custom: Malpractice law at the millenium". The Washington and Lee Law Review 57: 163.

Pfadenhauer, M. 2006. "Crisis or decline? Problems of legitimation and loss of trust in modern professionalism". Current Sociology 54: 565-578.

Richardson, G. 2001. "A tale of two theories: Monopolies and craft guilds in medieval England and modern imagination". Journal of the History of Economic Thought 23: 217-242.

Ritzer, G., D. Walczak. 1988. "Rationalization and the deprofessionalization of physicians". Social Forces 67: 1-22.

Rothman, R. A. 1984. "Deprofessionalization: The case of law in America”. Work and Occupations 11: 183-206.

Sabel, Ch. 2006. "Theory of a real-time revolution". Pp.106-157 in The Firm as a Collaborative Community: Reconstructing Trust in the Knowledge Economy. New York: Oxford University Press.

Salaburu, P. 2007. La Universidad en la Encrucijada: Europa y Estados Unidos. Madrid: Academia Europea de Ciencias y Artes.

Sciulli, D. 2005. "Continental sociology of professions today: Conceptual contributions". Current Sociology 53: $915-942$.

Scott, W. R. 1982. "Managing professional work: Three models of control for health organizations". Health Service Research 17: 213-240.

Shaban, S. 2007. "Multiple authorship trends in prestigious journals from 1950 to 2005". Saudi Medical Journal 28: 927-932.

Spellings, M. 2006. A Test of Leadership: Charting the Future of US Higher Education. Jessup, MD: U.S. Dept. of Education - Education Publications Center. 
Sullivan, W. M. 2005. Work and Integrity: The Crisis and Promise of Professionalism in America. San Francisco, CA: Jossey-Bass.

Unceta, A. 2011. "La educación superior en tránsito: ¿Es Bolonia la ruta idónea para la innovación?" ARBOR Ciencia, Pensamiento y Cultura 187: 1119-1131.

Vedder, R. 2011. "Time to make professors teach". The Wall Street Journal, 8 de junio.

Waters, M. 1989. "Collegiality, bureaucratization, and professionalization: A Weberian analysis". American Journal of Sociology 94: 945-972.

Charles Heckscher es catedrático de Organización de Empresas y Director del Center for Organizational Learning \& Transformation (COLT) de la Universidad de Rutgers en Nueva Jersey (Estados Unidos). Por más de cuarenta años su interés investigador se ha centrado en cuestiones de democracia en el trabajo y de relaciones colaborativas. Ha recibido numerosos premios de investigación en el ámbito del cambio organizativo y las relaciones laborales. Es autor, entre otros, de los libros The New Unionism, White-Collar Blues, Agents of Change o The Collaborative Enterprise.

Carlos Martín-Ríos es senior researcher en el Center for Organizational Learning \& Transformation (COLT) de la Universidad de Rutgers en Nueva Jersey (Estados Unidos) y Managing Director del Center for Management Innovation (CMI-Madrid). Su trabajo de investigación se sitúa en los ámbitos de la teoría de las organizaciones, la innovación organizativa y de gestión y el estudio de las relaciones inter-organizativas. Su más reciente línea de investigación explora la importancia de los sistemas colaborativos en entornos complejos como las universidades, los centros públicos de investigación o las empresas intensivas en conocimiento. 